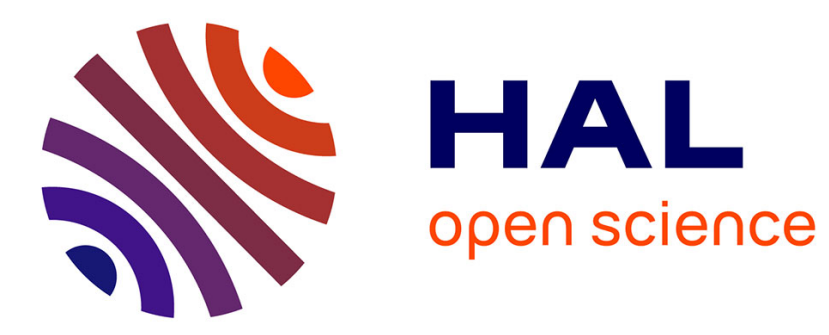

\title{
Interference tomography of the synoptic inhomogeneities and ocean bottom
}

A. Kazarova, L. Lyubavin, A. Nechaev

\section{To cite this version:}

A. Kazarova, L. Lyubavin, A. Nechaev. Interference tomography of the synoptic inhomogeneities and ocean bottom. Journal de Physique IV Proceedings, 1994, 04 (C5), pp.C7-1099-C7-1103. 10.1051/jp4:19945242 . jpa-00252930

\section{HAL Id: jpa-00252930 https://hal.science/jpa-00252930}

Submitted on 1 Jan 1994

HAL is a multi-disciplinary open access archive for the deposit and dissemination of scientific research documents, whether they are published or not. The documents may come from teaching and research institutions in France or abroad, or from public or private research centers.
L'archive ouverte pluridisciplinaire HAL, est destinée au dépôt et à la diffusion de documents scientifiques de niveau recherche, publiés ou non, émanant des établissements d'enseignement et de recherche français ou étrangers, des laboratoires publics ou privés. 


\title{
Interference tomography of the synoptic inhomogeneities and ocean bottom
}

\author{
A.Yu. KAZAROVA, L.Ya. LYUBAVIN and A.G. NECHAEV
}

Institute of Applied Physics, Russian Academy of Sciences, 46 Ulyanov St., 603600 Nizhny Novgorod, Russia

\begin{abstract}
The interference algorithm of the acoustic tomography of the ocean is discussed. In this method the synoptic inhomogeneities of the sound velocity field are restored by the perturbations in the intermode phase differences. The intermode phase difference, in its turn, is determined by the measured space distribution of the intensity (the interference structure) of the acoustic field. The interference method is also applied for determining the vertical profiles of sound speed, density and attenuation coefficient in the bottom. The possibility of using this method is numerically analysed for natural ocean conditions.
\end{abstract}

\section{INTRODUCTION}

The interference algorithm of the acoustic tomography of the ocean is proposed [1], where the synoptic inhomogeneities of the sound velocity field are restored by using the perturbations in the intermode phase differences. The intermode phase difference, in ito turn, is determined by measuring the space distribution of the intensity (the interference structure) of the acoustic field (solving the phase problem). Also, the interference method of determining the vertical profiles of sound speed, density and attenuation coefficient in the bottom by measuring the sound interference structure and using a priori evaluation of the bottom parameters is suggested. In the present paper the possibilities to solve the phase problem and to determine the bottom parameters for natural ocean conditions are numerically analysed.

1. Interference tomography of the synoptic inhomogeneities.

We consider an irregular underwater waveguide with the sound velocity profile varying horizontally. Let a harmonic sound source with power $P_{0}$ locate in the point $(x=0, y=$ 
$\left.=0, z=z_{s}\right)$, and the receiver - in the point $\left(x=a, y=0, z=z_{r}\right)$, where $x, y$ are the horizontal coordinates, $z$ is the vertical coordinate directed downwards. We assume that the vertical profiles of the sound velocity near the source and the receiver are equal to $c(z)$. We consider the bottom to be smooth, unabsorbing and homogeneous. We suppose that there are inhomogeneities in the acoustic trace between the source and the receiver which cause the synoptic variations of the sound velocity field $\delta c(x, y, z)$; there being no other types of inhomogeneities there.

Assuming that for all pairs of modes $\left|\varepsilon_{n m}\right| \ll 1$, where $\varepsilon_{n m}$ are perturbations in the intermode phase difference caused by synoptic inhomogeneities, we obtain for the intensity variation of the acoustic field in the adiabatic approximation [1]:

$$
\Delta I\left(a, z_{s}, z_{r}\right)=\tilde{I}\left(a, z_{s}, z_{r}\right)-I\left(a, z_{s}, z_{r}\right) \simeq \sum_{i=1}^{N-1} K_{i}\left(a, z_{s} ; z_{r}\right) \varepsilon_{i}
$$

where

$$
K_{i}\left(a, z_{s}, z_{r}\right)=-\frac{P_{0}}{a} \sum_{n=1}^{i} \sum_{m=i+1}^{N} \frac{\varphi_{n}\left(z_{s}\right) \varphi_{n}\left(z_{r}\right) \varphi_{m}\left(z_{s}\right) \varphi_{m}\left(z_{r}\right) \sin \left(\left(h_{n}-h_{m}\right) a\right)}{\sqrt{h_{n} h_{m}}}
$$

$\tilde{I}\left(a, z_{s}, z_{r}\right)$ is the intensity of the acoustic field in the receiver in the presence of inhomogeneities, $I\left(a, z_{s}, z_{s}\right)$ is the intensity in the receiver without inhomogeneities, $\varphi_{n}$ and $h_{n}$ are the eigenfunctions and horizontal wave numbers of modes in the unperturbed waveguide, $\varepsilon_{i}=\varepsilon_{i i+1}$ are the perturbations in the intermode difference of phases of two adjacent modes, $N$ is the number of modes. It may be shown that the case, when there are certain values of $\varepsilon_{n m}$ which are not small in comparison with unity is taken into account in the frames of such an a approximation by using the space Fourier filtration of the horizontal sound interference structure.

The problem of reconstruction of the intermode phase difference by the acoustic field interference structure is reduced to determining unknown parameters $\varepsilon_{n m}$ from $\mathrm{F}_{\mathrm{q}}$.(1).

The calculations are made for two characteristic types of the ocean underwater waveguide: a deep-sea waveguide with a canonical profile of the sound velocity and a subsurface waveguide with the linear profile of the sound velocity. As a perturbating inhomogeneity, cold (for the deep-sea channel) and warm (for the subsurface channel) synoptic eddies are considered. The parameters of the unperturbed waveguide acoustic modes are calculated with the aid of the mode program. In the process of modeling the phase problem, the influence of sea noises is taken into account. The accuracy of the reconstruction is estimated by the coefficient

$$
\gamma=\sqrt{\frac{\sum_{i=1}^{N-1}\left|\Delta \varepsilon_{i}\right|^{2}}{\sum_{i=1}^{N-1}\left|\varepsilon_{i}\right|^{2}}}
$$


where $\Delta \varepsilon_{i}$ is the difference between the true and the restored values of $\varepsilon_{i}$.

For "energetically rather weak" eddies (for all pairs of modes $\left|\varepsilon_{n m}\right| \ll 1$ ) the satisfactory restoration of $\varepsilon_{i}$ ( the accuracy $\gamma \leq 0,14$ ) in the subsurface channel may occur by a onedimensional (horizontal or vertical) interference structure. In the deep-sea channel the measurement of the two-dimensional interference structure is obligatory.

In the case of "energetically strong enough eddies" $\left(\Delta c_{i}=15-30 \mathrm{~m} / \mathrm{s}\right.$, where $\Delta c$ is the absolute maximum perturbation of sound velocity in the eddy), when the space filtering (permitting to single out a large-scale interference structure with $\left|\varepsilon_{n m}\right| \ll 1$ ), should be used, it is necessary to measure the two-dimensional interference structure in both types of channels for the satisfactory restoration $\bar{\varepsilon}_{i}$.

To illustrate the calculations performed, Fig.1 gives the results of solving the phase problem in a deep-sea channel. The calculation was made at $\Delta c=+15 \mathrm{~m} / \mathrm{s}$. In this case the most optimal for the restoration the interval of the numbers $42 \leq i \leq 65$ appeared. The case of the subsurface channel is given in Fig.2. Here the optimal for the restoration is the interval of numbers $54 \leq i \leq 70$.
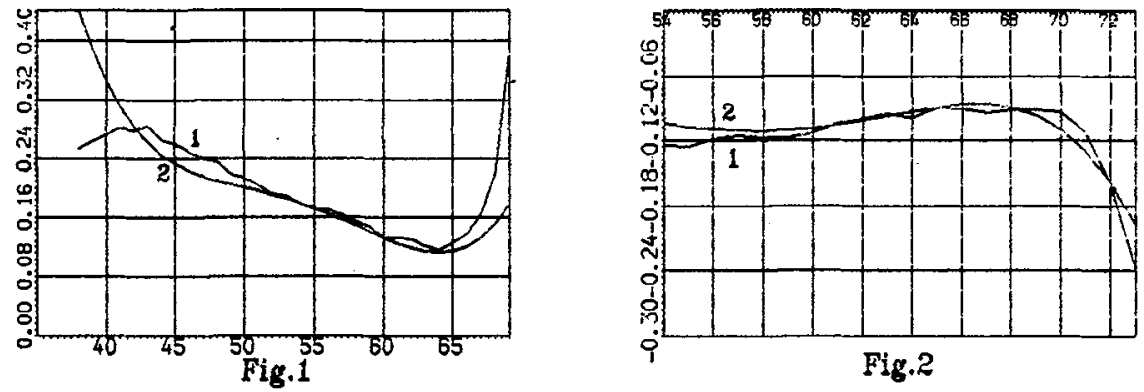

The variation of the noise intensity has shown that the satisfactory restoration of $\varepsilon_{i}$ $(\gamma \leq 0,12)$ is observed at the signal-to-noise ratio of the order or greater than $13 \mathrm{Db}$.

Thus, by measuring the acoustic field interference structure one may determine accurately enough the intermode phase difference ( at least for a certain interval of mode numbers ).

2. Interference tomography of the ocean bottom.

Let us consider an oceanic waveguide with jumps of the sound speed and density at the water $(0 \leq z \leq H)$ - bottom $(H \leq z)$ boundary. The $z$ axis is directed downward. Let the profile of the sound speed be $\tilde{c}=c(z)+\delta c$ and of the density be $\tilde{\rho}=\rho(z)+\delta \rho(z)$. Here, $c(z)$ and $\rho(z)$ are a priori evaluations of sound speed and density. The sound propagation will be described by an equation for the complex amplitude of sound pressure $p \cdot \exp (i \omega t)$

$$
\tilde{\rho} \nabla\left(\frac{\nabla \tilde{p}}{\tilde{\rho}}\right)+\frac{\omega^{2}}{\tilde{c}^{2}}(1-2 i \tilde{\gamma}) \tilde{p}=Q \delta^{2}\left(\vec{r}-\vec{r}_{0}\right) \delta\left(z-z_{0}\right)
$$


Where $\omega$ is the circle frequency, $\tilde{\gamma}=\omega(\lambda+2 \mu) / \tilde{\rho} \tilde{c}^{2} \simeq \gamma=\omega(\lambda+2 \mu) / \rho c^{2}, Q=\sqrt{8 \pi \rho_{0} c_{0} P}$, $P$ is the source power, $\rho_{0}$ and $c_{0}$ are the density and sound speed near the sonrce point $\left(\vec{r}_{0}=0, z_{0}\right)$. The magnitude $\tilde{\gamma} \omega / c$ is the attenuation coefficient in a homogeneous medium.

The formula relating the pressure field intensity with the variations (the differences from the basic values) of the bottom parameters is used as the initial one for reconstruction of the vertical profiles of the sound speed, density and attenuation coefficient. It is obtained in the following way. Expression for the pressure field intensity is written using the modes description . Average values in area under investigation or other a priori evaluations can be used as reference. The modes parameters (eigenvalues, eigenfunctions) are expressed through the bottom parameters by means of the perturbation theory. After that, formula is linearized for a short path, the path for which variations of amplitude and phase of modes are small. This linearization simplifies the formula.

Let us consider some results of numerical reconstruction of the sound attenuation coefficient in a homogeneous bottom by measuring the sound intensity in the case of random noise and unknown source power. Without the sound speed and density variation we can write the above-mentioned formula as

$$
\delta I\left(a, z, z_{0}\right)=\tilde{I}-I=P \int_{D}^{\infty} d z^{\prime} \gamma\left(z^{\prime}\right)\left[K_{1}\left(z^{\prime}, a, z, z_{0}\right)+K_{2}\left(z^{\prime}, a, z, z_{0}\right)\right]
$$

where

$$
\begin{gathered}
K_{1}\left(z^{\prime}, a, z, z_{0}\right)=-\frac{\omega^{2}}{c^{2}\left(z^{\prime}\right)} \frac{\rho_{0}}{\rho\left(z^{\prime}\right)} \sum_{n=1}^{N} \sum_{k=1}^{N} \frac{q_{n}\left(z, z_{0}\right) q_{k}\left(z, z_{0}\right)}{\sqrt{h_{n} h_{k}}} \frac{\varphi_{n}^{2}\left(z^{\prime}\right)}{h_{n}} \cos \left[\left(h_{n}-h_{k}\right) a\right], \\
K_{2}\left(z^{\prime}, a, z, z_{0}\right)=\frac{\omega^{2}}{c^{2}\left(z^{\prime}\right)} \frac{\rho_{0}}{\rho\left(z^{\prime}\right)} \sum_{n=1}^{N} \sum_{k=1}^{N} \frac{q_{n}\left(z, z_{0}\right) q_{k}\left(z, z_{0}\right)}{a \sqrt{h_{n} h_{k}}} \sin \left[\left(h_{n}-h_{k}\right) a\right] \\
{\left[g_{n}\left(z, z_{0}\right) \frac{\varphi_{n}^{2}\left(z^{\prime}\right)}{2 h_{n}^{2}}-2 \sum_{m \neq n} \frac{\varphi_{n}(z) \varphi_{m}\left(z_{0}\right)+\varphi_{m}(z) \varphi_{n}\left(z_{0}\right)}{h_{n}^{2}-h_{m}^{2}} \varphi_{n}\left(z^{\prime}\right) \varphi_{m}\left(z^{\prime}\right)\right] .}
\end{gathered}
$$

$\tilde{I}=|\tilde{p}|^{2} / 2 \rho_{0} c_{0}, q_{n}\left(z, z_{0}\right)=\varphi_{n}(z) \varphi_{n}\left(z_{0}\right), \varphi_{n}$ and $h_{n}$ are the eigenfunctions and the horizontal wave numbers in waveguide with caracteristics $c(z), \rho(z)$ and $\gamma=0, a=\left|\vec{r}-\overrightarrow{r_{0}}\right|$.

Supposing $\gamma(z)=\gamma=$ const, and taking into account the noise intensity we can represent equation (3) as

$$
I\left(a, z, z_{0}\right)=\xi\left(\tilde{I}\left(a, z, z_{0}\right)+I_{n s}\right)-\gamma_{1} Q_{1}\left(a, z, z_{0}\right)-\gamma_{2} Q_{2}\left(a, z, z_{0}\right),
$$

where the sound intensity $I$ in the base waveguide and the coefficients $Q_{i}=P_{0} \int d z^{\prime} K_{i}, i=$ $=1,2$ are calculated for the case of a source with fixed power $P=P_{0}, I_{n s}$ is the noise intensity, $\xi=P_{0} / P_{*}, P_{*}$ is unknown source power. Instead of the value $\gamma$ we have formally substituted 
the uncorrelated values $\gamma_{1}$ and $\gamma_{2}$. Numerical simulation of the $\gamma$ reconstruction was carried out on the base of formula (4).

We consider the following model: the sea depth is $295 \mathrm{~m}$, the sound speed in water is $1,483 \mathrm{~km} / \mathrm{s}$, the bottom sound speed is $1,750 \mathrm{~km} / \mathrm{s}$, the source depth is $75 \mathrm{~m}$ and the depth receiver is $55 \mathrm{~m}$. As noise intensity we consider random value $I_{n s}$ with a uniform distribution in the interval $\left[0,2\left\langle I_{n s}\right\rangle\right]$ and dispersion $\left\langle I_{n s}\right\rangle^{2} / 3$. Random values of noise intensity are added to the values of $\tilde{I}$ in the measuring points. In the numerical reconstruction we employed an integral equation solving programs based on Tikhonov's regularizing technique. The horizontal interval used for reconstruction was equal to $0,5 \mathrm{~km}-8 \mathrm{~km}$.

The dependences of reconstruction error $\eta=\left|\gamma-\gamma_{r}\right| / \gamma$ (where $\gamma_{r}$ is the value obtained in the reconstruction) on the attenuation coefficient (Fig.3) and the noise intensity $\zeta=$ 10. $\lg \left(I_{m} /\left\langle I_{n s}\right)\right)\left(I_{m}\right.$ is the maximum of the sound intensity) (Fig.4) without density and sound speed variations are shown. Graph 1 describe the reconstruction of $\gamma_{1}$, graph $2-\gamma_{2}$.

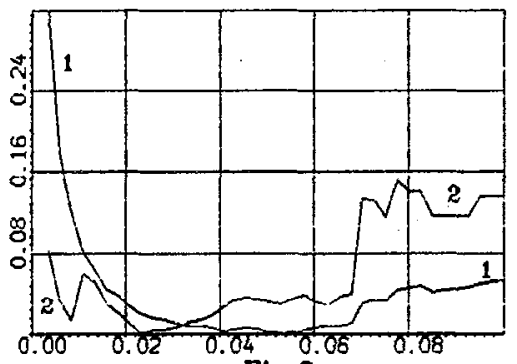

Fig.3

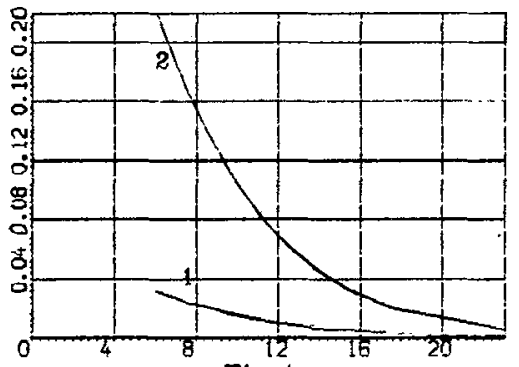

Fig.4

Numerical simulation showed the possibility of good-accuracy restoration of the sound attenuation coefficient for values of the signal-to-noise ratio about or larger $10 \mathrm{~dB}$ in the case of unknown source power.

References.

[1] Lyubavin L.Ya. and Nechaev A.G. Akust. zh. (1989) v.35. N4. p.703-710. 